\title{
A criança portadora de diabetes Tipo 1: implicações para Enfermagem
}

\author{
The child with Type I diabetes: implications for Nursing
}

El niño con diabetes Tipo I: implicaciones para la Enfermería

\section{Thamires Da Rosa Machado ${ }^{1}$, Alessandra da Silva Souza ${ }^{2}$, Jannaina Sther Leite Godinho Silva ${ }^{3}$, Eliara Adelino da Silva ${ }^{4}$, Geisa Sereno Velloso da Silva ${ }^{5}$, Adiel Queiroz Ricci ${ }^{6}$}

Como citar esse artigo. Machado, T. R. Souza, A. S; Silva, J. S. L G.; da Silva E. A.; da Silva, G. S. V.; Ricci, A. Q. A criança portadora de diabetes Tipo 1 : implicações para Enfermagem . Revista Pró-UniverSUS. 2021 Jul./Dez;; 12 (2) $32-38$.

\section{Resumo}

O presente estudo tem como objetivo avaliar, por meio de pesquisa a trabalhos já publicados, como se dá a participação do profissional da enfermagem no tratamento de diabetes tipo 1 em crianças. Trata-se de uma pesquisa de natureza qualitativa, descritiva, exploratória, que busca, por meio de revisão bibliográfica, familiaridade com o problema. A coleta de dados ocorreu por meio de plataformas, biblioteca Virtual em Saúde (BVS), Scientific Electronic Library Online (SciELO), Revista Brasileira de Enfermagem (REBEn) em língua portuguesa, além da Sociedade Brasileira de Diabetes do ano 2019 e o livro Wong Fundamentos da Enfermagem Pediátrica do ano 2014 como auxiliar, no período correspondente ao mês de março a outubro de 2020. Ao analisar os estudos pode-se observar que é relevante o conhecimento do profissional de saúde sobre o tema diabetes, para realizar assistência e orientação referente à doença, que necessita de cuidados especiais na rotina de forma vitalícia. Concluiu-se que é possível minimizar a dor e ansiedade referente à patologia, orientando o familiar do portador da doença ou a própria criança, que há métodos de aderir aos cuidados de formas mais dinâmicas, tais como orientações aos cuidadores sobre alimentação saudável, prática de exercício físico, auto aplicação de insulina e o rodízio de aplicação.

Palavras-chave: Enfermagem; Diabetes mellitus; Saúde da criança.

\begin{abstract}
The present study aims to evaluate, through research on previously published works, how the nursing professional participates in the treatment of type 1 diabetes in children. Methodology: This is a qualitative, descriptive, exploratory research that seeks to familiarize itself with the problem through the bibliographic review and, therefore, address the main themes on the subject. Data collection occurred through platforms, Virtual Health Library, SciELO in Portuguese, REBEn, in addition to the Brazilian Diabetes Society of the year 2019 and a book as an auxiliary, in the period corresponding to March to October 2020.When analyzing the studies, it can be seen that the knowledge of the health professional on the subject of diabetes is relevant to provide assistance and guidance regarding the disease, which requires special care in the routine for life. It was concluded that it is possible to minimize the pain and anxiety related to the pathology, advising the relative of the patient with the disease or the child himself, that there are methods of adhering to care in a more dynamic way, such as guidance to caregivers about food healthy, practice of physical exercise, self-application of insulin as well as the rotation of application.
\end{abstract}

Keywords: Nursing; Diabetes mellitus; Child Health.

Afiliação dos autores:

${ }^{1}$ Graduanda de Enfermagem, Universidade de Vassouras, Vassouras, Rio de Janeiro, Brasil. ORCID: https://orcid.org/0000-0002-7995-1444

${ }^{2}$ Enfermeira Mestre em Enfermagem pela Universidade de Vassouras. Professora Adjunta da Universidade de Vassouras, Rio de Janeiro, Brasil. ORCID: https://orcid.org/0000-00029009-9774

${ }_{3}^{3}$ Mestre em Enfermagem pela Universidade Federal Fluminense. Professora Adjunta da Universidade de Vassouras, Rio de Janeiro, Brasil. ORCID: https://orcid.org/0000-00028308-2093

${ }^{4}$ Enfermeira Mestre em Enfermagem pela Unifoa. Professora Adjunta da Universidade de Vassouras, Rio de Janeiro, Brasil. ORCID: https://orcid.org/0000-0003-4387-6662

${ }^{5}$ Enfermeira Mestre em Enfermagem pela UFRJ. Professora Adjunta da Universidade de Vassouras, Rio de Janeiro, Brasil. .com ORCID: https://orcid.org/0000-0003-0304-8010

${ }^{6}$ Doutorando em Estudos de Linguagem pelo programa de Estudos de Linguagem da Universidade Federal Fluminense, Professor Assistente da Universidade de Vassouras, Rio de

Janeiro, Brasil. ORCID: https://orcid.org/0000-0003-2761-2499.

* Email de correspondencia: thatarosa-10@hotmai.com

Recebido em: 22/01/21 Aceito em: 29/11/21 


\section{Resumen}

El presente estudio tiene como objetivo evaluar, a través de la investigación de trabajos publicados previamente, cómo se produce la participación de profesionales de enfermería en el tratamiento de la diabetes tipo 1 en niños. Es una investigación cualitativa, descriptiva, exploratoria que busca, a través de la revisión bibliográfica, el conocimiento del problema. La recolección de datos se realizó a través de plataformas, Biblioteca Virtual en Salud (BVS), Biblioteca Electrónica Científica en Línea (SciELO), Revista Brasileña de Enfermería (REBEn) en portugués, además de la Sociedad Brasileña de Diabetes del año 2019 y el libro Wong Fundamentos de Enfermería Pediátrica en 2014 como auxiliar, en el período correspondiente al mes de marzo a octubre de 2020. Al analizar los estudios, se puede apreciar que el conocimiento del profesional de la salud en el tema de la diabetes es relevante, para brindar asistencia y orientación sobre enfermedad, que necesita cuidados especiales de rutina de por vida. Se concluyó que es posible minimizar el dolor y la ansiedad relacionados con la patología, orientando al familiar de la persona con la enfermedad o al propio niño, que existen métodos de adhesión al cuidado de formas más dinámicas, como la orientación a los cuidadores sobre una alimentación saludable, ejercicio físico, autoaplicación de insulina y rotación de aplicación.

Palabras clave: Enfermería; Diabetes mellitus; Salud Infantil.

\section{Introdução}

Diabetes Mellitus (DM) é um quadro de hiperglicemia (elevação do açúcar no sangue), resultado da insuficiência da produção ou na secreção do hormônio insulina, desenvolvida no pâncreas pelas chamadas células beta. ${ }^{1}$

A diabetes é um importante problema de saúde pública, seu índice vem aumentando em diversas faixas etárias e em várias partes do mundo, atingindo proporções epidêmicas. ${ }^{2}$ O Brasil possui 16,8 milhões de adultos diabéticos, sendo o $4^{\circ}$ país no mundo com $8 \%$ a $9 \%$ da população acometida por diabetes, cuja prevalência maior é em mulheres do que em homens. Dados mostram, inclusive, que o Brasil ocupa a terceira posição com mais casos entre crianças e adolescentes. ${ }^{1}$

A cada ano, a quantidade de pessoas detectadas com diabetes cresce em números alarmantes, de forma que $40 \%$ dos brasileiros não sabem que são portadores da doença. Grande parte desse problema está exatamente ligada à rotina e, com o estresse e a agitação do dia a dia, as pessoas possuem hábitos que não são saudáveis. Com estilo de vida ruim, esse ritmo é perpetuado e a tendência é do aumento do número de pessoas acometidas. Os dados estatísticos estimam que existem 415 milhões de pessoas no mundo com DM, com projeção do aumento desse total para 642 milhões em $2040 .^{3}$

O diabetes tipo 1 causa quadro de hipoglicemia, $o$ que leva à necessidade de reposição de insulina exógena utilizada no tratamento. Desse modo, alguns cuidados são essenciais nesta doença, tais como a necessidade diária de aplicação de insulina, estar atento à oscilação na glicemia e a grande tendência a desenvolver cetoacidose e coma. ${ }^{4}$ Essa doença pode causar danos como: complicações no coração, nas artérias, nos olhos, nos rins e nos nervos e, em casos mais graves, leva ao óbito. Quando há elevação prolongada da glicemia, alguns sinais são detectáveis, como febre, vômitos, urinar em excesso, dor abdominal ou taquipneia, caracterizada pela aceleração do ritmo respiratório. ${ }^{5}$

A incidência da diabetes mellitus tipo 1 vem aumentando, especialmente na população infantil, e ocorre em crianças entre 5 à 6 anos, o que representa cerca de $5 \%$ a $10 \%$ da prevalência dos casos de diabetes. Segundo estimativas, $2 \%$ a $3 \%$ das crianças com DM1 (Diabete Mellitus tipo 1) morrem nos primeiros 10 anos após o diagnóstico, e $12 \%$ a $13 \%$ morrem nos 20 anos após o diagnóstico devido a complicações, como o excesso de episódios de hipo ou hiperglicemia. ${ }^{6}$

A evolução da doença crônica no Brasil é preocupante, já que é conceituado um grave problema de saúde pública, responsável por $63 \%$ dos óbitos no mundo. Deste modo, a pesquisa justifica-se pelo fato de que há grande necessidade de uma assistência adequada às crianças, enquanto pacientes portadores da doença, como um procedimento que lhes garanta uma qualidade de vida prolongada, elaborando rotinas com hábitos saudáveis, exercícios e uso da medicação correta, a fim de prevenir que as crianças tenham complicações em longo prazo, trazendo consigo certa independência nas aplicações de fármacos e orientando os responsáveis para que haja uma compreensão mais acertada sobre a doença. ${ }^{2}$

Observa-se, portanto, como problema, a ausência de cuidados prestados às crianças portadoras dessa doença crônica e de orientações necessárias aos responsáveis das mesmas, trazendo, desse modo, uma baixa adesão ao tratamento de qualidade. Desta forma, $\mathrm{o}$ presente estudo estrutura-se e fundamenta-se na maneira como o profissional da enfermagem pode auxiliar no tratamento de diabetes em crianças nas questões que envolvem o conhecimento dos pais acerca da doença e de como proceder, bem como o fornecimento de assistência adequada nas unidades básicas de saúde.

O objetivo geral desta pesquisa se dá em avaliar, por meio de pesquisa a trabalhos já publicados, como se dá a participação do profissional da enfermagem no tratamento de diabetes tipo 1 em crianças, tendo como 
objetivos específicos analisar as maneiras como ocorre a assistência familiar oferecida pela rede pública de saúde, e identificar as fragilidades dos cuidadores, a partir dos dados observados no estudo.

\section{Metodologia}

Trata-se de uma pesquisa de natureza qualitativa, descritiva, exploratória, que busca, por meio da revisão bibliográfica, familiaridade com o problema e, por conseguinte, abordar os principais temas sobre o assunto.

A pesquisa qualitativa baseia-se em característica subjetiva, tornando-se importante para o entendimento da realidade humana, das dificuldades vivenciadas, das atitudes e dos comportamentos dos sujeitos envolvidos. Representa um suporte teórico essencial, sem utilizar números, e sim narrativas. ${ }^{7}$

Existem três importantes momentos que devem ser levados em consideração quando se opta por esta abordagem qualitativa: a fase de exploração da pesquisa; a análise dos resultados finais e a elaboração do texto final. $^{7}$

O estudo exploratório possibilita ao pesquisador captar conhecimentos e evidências teóricas, a partir de investigações de determinadas hipóteses para desenvolver, posteriormente, uma pesquisa descritiva ou ainda experimental. ${ }^{8}$

Pesquisa descritiva tem objetivo de descrever as características de uma população, um fenômeno ou experiência para o estudo realizado, fazendo o estudo, a análise, o registro e a interpretação dos fatos do mundo físico, sem sua manipulação ou interferência. Ele deve apenas descobrir a frequência com que o fenômeno ocorre ou como se estrutura dentro de um determinado sistema, método, processo ou realidade operacional. ${ }^{8}$

Como critério de inclusão, foram seelcionados artigos que apresentassem as palavras chaves: "Enfermagem; Diabetes mellitus; Saúde da Criança" e, como critério de exclusão, aqueles quenão se adequassem ao tema e que não contemplassem o conceito chave. As bases de dados foram a biblioteca Virtual em Saúde (BVS), Scientific Electronic Library Online (SciELO), Revista Brasileira de Enfermagem (REBEn) em língua portuguesa, além da Sociedade Brasileira de Diabetes do ano 2019, bem como o livro Wong Fundamentos da Enfermagem Pediátrica do ano 2014. A seleção dos artigos contempla aqueles publicados nos últimos 10 anos. Após a leitura e resumos, 30 artigos foram selecionados, dos quais apenas 21 estavam dentro do propósito de estudo da pesquisa. O período de coleta dos dados ocorreu no período de março a outubro de 2020, durante o qual as obras escolhidas foram analisadas criteriosamente.

\section{Resultados e Discussão}

Através da busca na base de dados, foi realizada a leitura e a interpretação do material encontrado. Da análise emergiram duas unidades temáticas: As crianças com demandas de cuidados contínuos;o diagnóstico de diabetes e o enfermeiro educador de um cuidado inovador para a crinaça com diabetes e sua família.

\section{As crianças com demandas de cuidados contínuos}

As crianças com demandas de cuidados contínuos envolvem familiares cuidadores na prestação de cuidados que atendam às necessidades de alimentação, oxigenação, eliminação, conforto, segurança e bemestar. ${ }^{9}$

O diabete melito é uma doença crônica e considerada um dos maiores problemas de saúde pública, se iniciando de uma forma gradual e pode apresentar uma longa ou indefinida duração, o que requer um cuidado contínuo em nível domiciliar ou hospitalar. As doenças crônicas exige uma necessidade constante da intervenção de profissionais de saúde, devido aos problemas ocasionados pela doença. Dependendo do tipo da doença crônica na infância, ela pode geram limitação de alguma função, dependência de medicamentos, alimentação especial, necessidade de dispositivo tecnológico ou de assistência direta em serviços de saúde. Desse modo, a mesma passou a ser denominada de Crianças com Necessidades Especiais de Saúde.$^{10}$

CRIANES é uma expressão adotada para designar um conjunto de crianças que apresentam condições crônicas, físicas, de desenvolvimento, comportamental ou emocional, requerendo assim uma maior utilização dos serviços de saúde e atendimento por diferentes profissionais das mais diversas especialidades, incluindo a enfermagem. ${ }^{11}$

No Brasil, segundo as demandas de cuidados, as CRIANES são classificadas em seis tipos. O primeiro, de desenvolvimento, inclui aquelas com disfunção neuromotora muscular, limitações funcionais e incapacitantes, em estimulação de desenvolvimento funcional. No de cuidados tecnológicos, estão as crianças em uso de dispositivos mantenedores da vida, como gastrostomia, traqueostomia, colostomia etc. No de cuidados medicamentosos, estão as que fazem uso contínuo de fármacos, tais como cardiotônicos, anticonvulsivantes etc. No de cuidados habituais modificados, a criança precisa de tecnologias adaptativas nos cuidados cotidianos e nas atividades de vida diária para locomover-se, alimentar-se, arrumar-se, no uso de toalete etc. No de cuidados mistos, há uma combinação de uma ou mais demandas, excluindo-se a tecnológica. No de demandas de cuidados clinicamente complexos, há uma combinação de todas as anteriores incluindo o 
manejo de tecnologias de suporte de vida. ${ }^{11}$

Desse modo, o estudo revelou que a que a criança portadora de diabetes e sua família é acometida por uma gama de sentimentos que precisam ser entendidos e trabalhados. O desconhecimento sobre a doença e suas complicações podem gerar momentos de intenso sofrimento, medo, preocupações, limitações e incertezas.

Neste sentido identificamos que há profissionais que possuem um olhar mais abrangente para as crianças portadoras de doenças crônicas e que novos modelos de cuidados precisam ser inseridos em todas as fases da doença principalmente no enfrentamento do diagnóstico.

\section{O diagnóstico de diabetes}

A tabela abaixo mostra o percentual de ocorrência de diabetes no Brasil, de acordo com o tipo e faixa etária:

Tabela 1. Percentual de diabéticos no Brasil com base em faixa etária e tipo ocorrente da doença.

\begin{tabular}{|c|c|c|}
\hline $\begin{array}{c}\text { TIPO DE } \\
\text { DIABETES }\end{array}$ & $\begin{array}{l}\text { \% ACOMETIDO } \\
\text { PELA DOENÇA }\end{array}$ & FAIXA ETÁRIA \\
\hline $\begin{array}{c}\text { Diabetes Mellitus } \\
\text { Tipo } 1 .\end{array}$ & $\begin{array}{c}\text { Acomete } 5 \text { a } 10 \% \\
\text { dos pacientes com } \\
\text { DM. }\end{array}$ & $\begin{array}{l}\text { 5-7 anos e na puber- } \\
\text { dade }\end{array}$ \\
\hline $\begin{array}{c}\text { Diabetes Mellitus } \\
\text { tipo } 2\end{array}$ & $\begin{array}{l}\text { Responsável por } \\
90-95 \% \text { dos casos. }\end{array}$ & $\begin{array}{l}\text { A faixa etária de } \\
\text { idade de início é } \\
\text { variável, embora } \\
\text { seja mais frequente } \\
\text { após os } 40 \text { anos. }\end{array}$ \\
\hline
\end{tabular}

Fonte: Adaptado ${ }^{1}$.

O diagnóstico do tipo de diabetes será definido com o estado clínico geral e dos resultados de exames, sendo necessários alguns exames para detecção, como:

- Teste de glicemia plasmática em jejum: mede a glicose no sangue após pelo menos 8 horas de jejum; ${ }^{12}$

- Teste oral de tolerância à glicose ((TOTG): previamente à ingestão de $75 \mathrm{~g}$ de glicose dissolvida em água, coleta-se uma amostra de sangue em jejum para determinação da glicemia coleta-se outra, então, após 2 horas da sobrecarga oral; ${ }^{12}$

- Hemoglobina Glicada (HbA1c): avalia os níveis médios da glicose sanguínea nos ultimos 2 ou 3 meses. ${ }^{12}$

Existem quatro tipos de diabetes, o tipo 1, seguido pelo diabetes tipo 2, diabetes gestacional e o Diabetes Latente Autoimune do Adulto (LADA):
Tabela 2. Os critérios laboratoriais para diagnóstico de diabetes, adotados pela Sociedade Brasileira de Diabetes (2019) .

\begin{tabular}{|c|c|c|c|}
\hline SITUAÇÃO & $\begin{array}{c}\text { GLICOSE } \\
\text { EM } \\
\text { JEJUM } \\
(\mathrm{mg} / \mathrm{dL})\end{array}$ & $\begin{array}{c}\text { GLICOSE } 2 \\
\text { HORAS } \\
\text { APÓS } \\
\text { SOBRECARGA } \\
\text { COM } 75 \text { g DE } \\
\text { GLICOSE } \\
\text { (mg/dL) }\end{array}$ & $\begin{array}{c}\text { HBA1C } \\
(\%)\end{array}$ \\
\hline $\begin{array}{c}\text { Normoglice- } \\
\text { mia }\end{array}$ & $<100$ & $<140$ & $<5,7$ \\
\hline $\begin{array}{c}\text { Pré-Diabetes } \\
\text { ou risco } \\
\text { aumentado } \\
\text { para DM }\end{array}$ & $\begin{array}{c}\geq 100 \mathrm{e}< \\
126\end{array}$ & $\geq 140 \mathrm{e}<200$ & $\begin{array}{l}\geq 5,7 \text { e }< \\
6,5\end{array}$ \\
\hline $\begin{array}{l}\text { Diabetes } \\
\text { estabelecido }\end{array}$ & $\geq 126$ & $\geq 200$ & $\begin{array}{l}\geq 200 \text { com } \\
\text { sint o mas } \\
\text { inequívocos } \\
\text { de hipergli- } \\
\text { cemia }\end{array}$ \\
\hline
\end{tabular}

Fonte: Adaptado ${ }^{2}$.

- Tipo 1: Sua fisiopatologia não é totalmente conhecida, ela está relacionada, além da predisposição genética, a fatores ambientais que desencadeiam a resposta autoimune. Possui duas formas, sendo o imunomediado, que resulta da destruição autoimune das células $B$, iniciando-se na infância e adolescência, podendo surgir em adultos de qualquer idade. Também se apresenta como tipo 1 idiopático, refere-se a forma rara da doença, que não tem fator desconhecido. Caracteriza-se por distúrbio crônico do metabolismo, por uma deficiência parcial ou completa da produção do hormônio insulina, consequente da destruição das células beta do pâncreas, causado geralmente de processo autoimune ou idiopático. Atinge as faixas entre 5-7 anos e na puberdade, sem preferência por sexo; ${ }^{13}$

- Tipo 2: É a resistência de insulina, onde o corpo não produz mais a mesma quantidade suficiente para controlar a taxa de glicemia, mais comum em indivíduos a partir da quarta década de vida. Tratase de uma doença multifatoriais, está relacionado à obesidade, hipertensão, presença de níveis elevados de lipídios (gorduras) no sangue hábitos alimentares ricos em gordura, sal, carboidratos ou açúcar, sedentarismo, histórico familiar e idade avançada, sendo a forma mais comum da doença; ${ }^{13}$

- Diabetes Gestacional: adquirida na gestação, neste período a placenta é uma fonte importante de hormônios que reduzem a ação da insulina, responsável pela captação e utilização da glicose pelo corpo o pâncreas aumenta a produção de insulina para compensar 
este quadro de resistência à sua ação. Em algumas mulheres, entretanto, este processo não ocorre e elas desenvolvem a doença, caracterizada pelo aumento do nível de glicose no sangue. Os sintomas são comuns aos outros tipos de diabetes; ${ }^{13}$

- Diabetes Latente Autoimune do Adulto (LADA): tipo novo de diabetes, que ainda está em estudo, sendo uma doença com deficiência de insulina por destruição progressiva dos ilhéus pancreáticos, sendo desenvolvida no adulto, representando 2 a $12 \%$ dos doentes com diabetes mellitus tipo $2 .{ }^{14}$

Diabetes tipo 1 é uma das mais frequentes doenças crônicas da infância e da adolescência, sendo a causa desconhecida. Sua incidência vem aumentando nas últimas décadas, em particular em crianças menores de 5 anos, de forma que se tornou a quarta causa de óbito no país e a segunda doença crônica mais comum nesta faixa. Seu tratamento é continuo e vitalício e, além de fazer uso da insulina, há necessidade de mudanças no estilo de vida, como a prática regular de exercício físico, dieta adequada, cuidados com membros inferiores, apoio psicológico e social. ${ }^{15}$

$\mathrm{Na}$ infância, segue-se o mesmo diagnóstico que no adulto, observar os sinais como polifagia, poliúria, polidipsia, obesidade e hiperglicemia. O diagnóstico deve ser feito através do nível plasmático de glicose em jejum $>126 \mathrm{mg} / \mathrm{dL}(>7,0 \mathrm{mmol} / \mathrm{L})$, nível aleatório de glicose $\geq 200 \mathrm{mg} / \mathrm{dL}(\geq 11,1 \mathrm{mmol} / \mathrm{L}$ ) $\mathrm{Hb}$ glicosilada $(\mathrm{HbA} 1 \mathrm{c}) \geq 6.5 \%$ e, algumas vezes, teste oral de tolerância à glicose. ${ }^{2} \mathrm{O}$ tratamento começa com a avaliação do controle metabólico, a monitoração da glicemia capilar realizada pela punção de ponta de dedo, sendo feita em diferentes horários do dia. As crianças não aceitam ser furadas muitas vezes no dia, sendo assim dificultoso realizar a punção na ponta do dedo, antebraço ou mão. ${ }^{5}$

Entretanto é necessário dialogar sobre todas as fases da doença desde o diagnóstico na atenção terciária ao momento de preparação de alta na qual o enfermeiro assume o seu papel social de educador deste cuidado inovador para a criança e sua família.

\section{O Enfermeiro educador de um cuidado inovador para a criança com diabete e sua família}

Crianças possuem suas particularidades e necessidades de acordo com sua idade. Logo após o diagnóstico, sua família modifica seu costumes e a alimentação da casa, normalmente, evitando o excesso de açúcar, salgadinhos, refrigerantes, entre outros, fazendo que o cliente adote hábitos saudáveis que poderão ser mantidos ao longo da infância e da adolescência ${ }^{5}$. A criança, por sua vez, pode não demonstrar sinais de hipoglicemia ou hiperglicemia, e cabe ao cuidador ter um olhar holístico para criança, e estar atento aos questionamentos e ações dessa criança, quando estiver com feição triste, suor em excesso, vertigens, sonolência, tremores e fraqueza, todos são sinais de hipoglicemia. Em casos de hiperglicemia, os sintomas são aumento da sede e a vontade de urinar frequente. ${ }^{5}$

A equipe deenfermagem possui o papelimportante no cuidado, pois ela vai criar planos de cuidados para a criança e a família. No entanto, a educação em saúde é voltada aos domínios e habilidades necessárias ao tratamento, sendo eles emocional, alimentar, prática de exercícios e orientações sobre aplicação de insulina. O cuidado de enfermagern pode ser baseado no sistema da North American Nursing Diagnost Assocíation (NANDA), que se configura como mais comumente aceito e define o diagnóstico como o processo de identificação, intervenção e julgamento dos problemas de saúde do indivíduo, família ou comunidade. ${ }^{16}$

Estudos ressaltam que um diálogo eficaz entre enfermeiro e cuidador gera uma boa assistência, pois identifica as fragilidades e torna o cuidado mais dinâmico. ${ }^{14} \mathrm{O}$ profissional de saúde deve orientar a família sobre os direitos que a pessoa portadora da doença crônica possui, tais como fornecimento de insumo, insulina e aparelho de HGT para verificação da glicemia capilar, de forma gratuita. A Lei 11.347/06 determina que os portadores de diabetes recebam do SUS, gratuitamente, os medicamentos e materiais necessários para o tratamento e a monitoração da glicemia capilar. ${ }^{17}$

As Redes de Atenção à Saúde (RAS), configuram organizações poliárquicas de serviços de saúde vinculados entre si e em prol de uma única missão, com oferta de atenção contínua e integral, na qual a Atenção Primária à Saúde (APS) é a coordenadora. Além do mais, o Ministério da Saúde definiu no Brasil como uma das linhas de cuidados prioritários na atenção às pessoas com doenças crônicas, trazendo consigo promoção da saúde através de serviços prestados pela RAS, como traços na identificação de riscos, apoio nas decisões, sistema de informação clínica e bem estar pessoal. A criança com diabetes traz um grande desafio, pois ela tem uma complexidade no cuidado. Porém, ela deve receber cuidado e assistência individuais através de relações nos serviços públicos de saúde. ${ }^{17}$

A literatura aborda sobre a criança ter uma alimentação saudável, realizar prática de exercício físico, seguir o tratamento corretamente, receber os cuidados necessários e fazer com que a família entenda sobre a importância do cuidado necessário. Estar atenta aos sinais de hiperglicemia e hipoglicemia a mudança no estilo de vida, podendo ter uma rotina normal. E no âmbito escolar requer cuidados com a alimentação, os sinais que o corpo dá referentes a oscilação de glicemia. Caso a criança entenda sobre a patologia e queira, o enfermeiro deve explicar sobre o autocuidado e criar métodos para facilitar o tratamento de acordo com a LEI 
$\mathrm{N}^{\circ}$ 16.925, de 16 DE JANEIRO de 2019 que discorre em seu Artigo $2^{\circ}-\mathrm{O}$ estabelecimento de ensino, creche ou similar, deverá capacitar seu corpo docente e equipe de apoio para acolher a criança e o adolescente portador de deficiência ou doença crônica, propiciando-lhe a integração a todas as atividades educacionais e de lazer que sua condição pessoal possibilite. 16

Proporcionar capacitação profissional sobre as doenças crônicas como diabetes, diagnóstico, sinais, sintomas, tratamento, e cuidados após o diagnóstico, pode controlar a incidência de diabetes, assim como realizar demonstrações de como se faz a verificação de glicemia capilar, a aplicação de insulina e o descarte de material. ${ }^{15} \mathrm{O}$ enfermeiro deve capacitar os profissionais da unidade de saúde, realizando uma boa educação em saúde na comunidade, com ações educativas voltadas para reorientar sobre os cuidados com pacientes diabéticos e ajudar no enfrentamento do processo saúdedoença. Os profissionais da unidade devem estar atentos aos sinais de hipo e hiperglicemia e orientar a família do portador da doença. ${ }^{18}$

Cabe ao profissional de saúde conversar com o portador da doença e observar se este possui conhecimento e entendimento suficientes sobre a diabetes e, com isso, verificar se ela deseja aprender a realizar seu auto cuidado. Mesmo a criança realizando a aplicação sozinha, os pais e/ou o cuidador devem supervisionar e orientar. ${ }^{19}$

A implementação de um plano de tratamento irá facilitar a vida da criança e do cuidador no processo do tratamento. Com isso, aspectos técnicos relacionados a monitorização da glicemia capilar, nutrição balanceada, adequadas às necessidades de crescimento $\mathrm{e}$ desenvolvimento, ajuste de dose de insulina, integração social em locais de convívio com outras crianças, manejo de situações nas quais a criança rejeite, alerta em relação à atenção aos irmãos não diabéticos; suporte ao cuidador e capacitação dos profissionais da unidade básica. ${ }^{5}$

De acordo com a literatura, o enfermeiro é educador de um cuidado inovador para a criança com diabetes e sua família, sendo necessário uma boa comunicação entre enfermeiro e paciente para o alcance de metas e bons resultados no cuidado. Será necessária a avaliação do enfermeiro com este paciente e cuidador, identificando de problemas, distúrbios na saúde, suas percepções dos problemas e compartilhamento informações para planejar as estratégias para alcançar as metas propostas, em comum acordo. ${ }^{3}$

Criar uma forma de cuidar descontraída e divertida pode causar menos dano à criança, pois ela possui limitações, tais como medo, tensão e dor, e criar técnicas para reduzir tais situações é essencial, elaborando formas descontraídas e baseadas em brincadeiras. De acordo com a resolução 546/2017, a enfermagem pode utilizar a técnica de brinquedo terapêutico na assistência, o que reduz o medo o e a dor da criança no ato da aplicação. ${ }^{20}$

Vale a pena ressaltar que o uso do brinquedo terapêutico está sendo inserido na prática assistencial às crianças e, com ela, alcançados inúmeros resultados positivos. O brinquedo terapêutico é classificado em três tipos: dramático, capacitador de funções fisiológicas, e institucional e/ou preparatório. O dramatizador é aquele em que a criança externaliza seus sentimentos fazendo uso de bonecos. O capacitador de funções fisiológicas é aquele em que a criança busca melhorar sua condição. Já o instrucional é utilizado para educar a criança a respeito do tratamento, a fim de fazê-la entender, geralmente utilizado na hospitalização de crianças. ${ }^{21}$

Considerando que a infância é uma fase de descobertas, brincadeiras e descontração, o brinquedo terapêutico institucional será uma maneira de mostrar à criança uma forma menos invasiva e traumática mediante ao tratamento de diabetes, promovendo um método mais educativo e explícito para realização da autoaplicação de insulina e clareza na adesão do tratamento. ${ }^{21}$

Nesta perspectiva, nota-se que o adoecimento por diabetes envolve o processo de ensino-aprendizagem de um um cuidado familiar inovador conduzido pelo enfermeiro, e envolve vários momentos do ensinar e do fazer, que incluem a explicação, observação, execução, supervisão, colaboração e avaliação do desempenho na realização de novos cuidados. ${ }^{16}$

A insulina basal é utilizada como tratamento rotineiro da DM tipo 1, as doses a serem administradas são indicadas pelo médico, os locais para aplicação de insulina devem manter um rodizio para não causar edemas ou caroços lipodistrofia. A alimentação da criança deve ter aporte calórico e nutricional adequado às necessidades individuais. ${ }^{5}$ Para uma criança é doloroso ter que enfrentar a realidade desta doença, pois modifica a infância, os pensamentos, hábitos e desejos, criando outro estilo de vida com rotinas, limites e restrições. ${ }^{6}$

A insulina é um hormônio que administra a quantidade de açúcar no sangue, sendo absorvida por alimentos, deste modo, o diabetes é uma doença em que o corpo não produz insulina ou há uma deficiência em utilizar corretamente a insulina que produz, fazendo com que a pessoa não consiga usar a glicose adequadamente. $\mathrm{O}$ alto nível de glicose no sangue leva a hiperglicemia, e esse fator por longo prazo pode acarretar em danos em órgãos, vasos sanguíneos e nervos. ${ }^{2}$

A aplicação de insulina é necessária para um tratamento eficaz e contínuo, porém a administração e constância do medicamento causam impacto na criança e seus familiares. O enfermeiro e o cuidador podem disponibilizar a oportunidade da criança aprender monitorar a glicose e aplicação da insulina, criando métodos educativos menos invasivos $\mathrm{e}$ doloridos, tornando os processos mais descontraídos e aceitáveis. ${ }^{19}$ 
O profissional de saúde deve orientar a família aos pequenos cuidados, como a utilização da insulina e seus insumos. A aplicação de insulina é um processo doloroso tanto para os pais quanto a criança, e cabe ao profissional ensinar métodos para tornar essa situação mais descontraída. Deve-se realizar o rodízio em locais de aplicação de insulina, tais como, braços, glúteos, coxas e abdome. O descartar de seringas, agulhas lancetas, tiras de glicemia devem ser colocadas em descarpack fornecido pela unidade, em casos onde não se tenha, o local ideal são garrafas plásticas e encaminhá-los para um Unidade de Saúde próxima. ${ }^{16}$

\section{Conclusão}

O estudo revelou que a diabetes mellitus é uma das doenças crônicas mais comuns na infância e uma das que mais exigem adaptações nos âmbitos fisico, psicológico e social, tanto por parte da criança como da familia. Neste sentido, é importante dialogar sobre a doença, promovendo prevenção e promoção da saúde. Tanto os portadores da patologia quanto os que não adquiriram, precisam ter precaução ao fazer uso de alimentos ricos em açúcares e gorduras, sendo necessária a prática cotidiana de exercicios fisicos.

Com relação aos pacientes com diabetes, suas vidas devem ser levadas de uma forma diferente de antes do diagnóstico, tendo rotinas de monitoração de glicemia, dieta, prática de exercício e tratamento com insulina.

Desse modo, cabe ao profissional da sáude favorecer uma qualidade de vida e bem estar, orientado a familia e o paciente sobre os cuidados necessários em ambos os aspectos.

\section{Referências}

1. Oliveira MDF, Damo NG, Lopes C, Raitz IW, Pereira L. A participação da extensão na resolução de caso de criança com Diabetes Mellitus Tipo 1. Extensio Rev Eletrônica Extensão, 17 (35), 2020, p. 96-107.

2. Sociedade Brasileira de Diabetes. Diretrizes da sociedade brasileira de diabetes 2019-2020. Clannad editora científica , 2019.

3. Araújo ESS, Silva L de F da, Moreira TMM, Almeida PC de, Freitas MC de, Guedes MVC. Nursing care to patients with diabetes based on King's Theory TT - Cuidado de enfermagem ao paciente com diabetes fundamentado na Teoria de King TT - Cuidado de enfermería al paciente con diabetes justificado en la Teoría de King. Rev Bras Enferm [Internet], 71 (3), 2018, p. 1092-8.

4. Pires AC, Chacra AR. A Evolução Da Insulinoterapia no Diabetes Melito Tipo 1. Arq Bras Endocrinol Metabol., 52 (2), 2008, p. 268-78.

5. Calliari LEP, Monte O. Abordagem do diabetes melito na primeira infância. Arq Bras Endocrinol Metabol. 2008;52(2):243-9.

6. Pennafort VP dos S, Queiroz MVO, Nascimento LC, Guedes MVC. Rede e apoio social no cuidado familiar da criança com diabetes. Rev Bras Enferm. 2016;69(5):912-9.

7. Ferreira CAL. Pesquisa Quantitativa e Qualitativa: Perspectivas para o Campo da Educação. Rev. Mosaico.2015;8(2):173-182.
8. MINAYO, Maria Cecília de Souza. O Desafio do Conhecimento. Pesquisa Qualitativa em Saúde. 12 ed, São Paulo - SP:Hucitec, 2010.

9. Góes FGB, Cabral IE. Discursos sobre cuidados na alta de crianças com necessidades especiais de saúdeRev Bras Enferm [Internet]. 2017;70(1):15461. DOI: http://dx.doi.org/10.1590/0034-7167-2016-0248.

10. Souza MHN, Nóbrega VM, Collet N. Rede social de crianças com doença crônica: conhecimento e prática de enfermeiros. Rev. Brasileira de Enfermagem. 2020;73(2):1-8.

11. Cabral IE, Moraes JRMM. Cuidadores familiares articulando a rede social de uma criança com necessidades especiais de saúde. Rev Bras Enferm [Internet]. 2015[cited 2016 Apr 07];68(6):769-76. Available from: http:// www.scielo.br/ pdf/reben/v68n6/en_0034-7167-reben-68-06-1078.pdf;

12.Marilyn J.Hockenberry ,David Wilson. Wong Fundamentos da Enfermagem Pediátrica. 9a ed. Rio De Janeiro : Elsevier; 2014.

13. Gross JL, Silveiro SP, Camargo JL, Reichelt AJ, Azevedo MJ de. Diabetes Melito: Diagnóstico, Classificação e Avaliação do Controle Glicêmico. Arq Bras Endocrinol Metabol. 2002;46(1):16-26.

14.Alves D, Kachan B, Carboni C, Mariano P, Silva R. LADA numa Unidade Integrada de Diabetes. Med Interna (Bucur). 2016;23(4):22-5.

15. Brito TB de, Sadala MLA. Juvenile diabetes: The family's experience with diabetic adolescents and pre-adolescents. Cienc e Saude Coletiva. 2009;14(3):947-60. 